\title{
Effect of charge carriers on the barrier height for vacancy formation on $\ln P(110)$ surfaces
}

\author{
U. Semmler, Ph. Ebert, ${ }^{\text {a) }}$ and K. Urban \\ Institut für Festkörperforschung, Forschungszentrum Jülich GmbH, 52425 Jülich, Germany
}

(Received 23 February 2000; accepted for publication 9 May 2000)

\begin{abstract}
We determine the energy barrier height for the formation of positively charged phosphorus vacancies in $\operatorname{InP}(110)$ surfaces using the rate of formation of vacancies measured directly from scanning tunneling microscope images. We found a barrier height in the range of $1.15-1.21 \mathrm{eV}$. The barrier height decreases with increasing carrier concentration. These results are explained by a charge separation during the vacancy formation process. (C) 2000 American Institute of Physics.
\end{abstract} [S0003-6951(00)02027-1]

Point defects affect to a large degree the electronic properties of semiconductors and thus their applicability in electronic devices. The extent to which point defects can influence the properties of a semiconductor depends, however, not only on the electronic structure of the defects, but also very sensitively on their concentrations. Therefore, it is highly desirable to determine the factors governing the formation of defects. Such knowledge would open the possibility to predict specific conditions for the formation of a particular defect and ultimately it may become even possible to design materials on the atomic level. However, this task turned out to be very complicated. On the one hand it is well known that in case of thermal equilibrium the concentrations of defects are controlled by their formation energies (i.e., the energy differences between the initial and final states). On the other hand, under nonequilibrium conditions the concentrations of defects are to a large degree governed by the barrier heights for their formation. Although most semiconductor devices are grown under kinetic rather than equilibrium conditions, little is known about the barrier heights for the formation of defects on semiconductors surfaces. In fact the formation of surface defects and subsequent incorporation as bulk defects have not received much attention despite their potential influence on semiconductor devices. Up to now most work concentrated on growth related diffusion and incorporation barriers. ${ }^{1.2}$ This may be partly due to the difficulty to measure barrier heights for surface defect formation and partly due to the very large computational effort necessary to determine the exact path and the corresponding barrier height.

In this letter we demonstrate the determination of the energy barrier height for the formation of positively charged phosphorus $(\mathrm{P})$ vacancies on $\operatorname{InP}(110)$ surfaces by directly observing the vacancy formation using scanning tunneling microscopy measurements. We identify the charge carrier system as a factor affecting the height of the energy barrier and quantify the effect of the Fermi energy position on the barrier. The data indicate that a charge separation during vacancy formation affects the barrier height.

For the experiments we investigated $p$-doped InP single

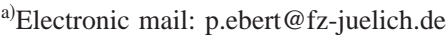

crystals with carrier concentrations of $5 \times 10^{17}(\mathrm{Cd}), 1.1$ $\times 10^{18}(\mathrm{Cd})$, and $8 \times 10^{18}(\mathrm{Zn}) \mathrm{cm}^{-3}$. The crystals were cleaved in ultrahigh vacuum along a (110) cleavage plane to expose clean and defect free surfaces. Scanning tunneling microscopy (STM) images reveal indeed only very few defects on the surfaces directly after cleavage. With increasing time the concentration of defects increases. Figure 1(a) and 1(b) show two constant current STM images obtained 3.2 and $56.6 \mathrm{~h}$ after cleavage, respectively. We found two types of defects. The bright localized contrast features arise from dopant atoms (they scale with the carrier concentration) and are not of interest here. Their properties have been described in detail before. ${ }^{3}$ Here we focus on the dark contrast features. The dark contrast features are indicative of a positive charge of the underlying defects as discussed in detail in Ref. 4. The defects can be identified on basis of a high resolution image obtained on the same surface [Fig. 1(c)]. The contrast in the occupied states is that of a $\mathrm{P}$ monovacancy, ${ }^{4,5}$ which is on $p$-doped $\operatorname{InP}(110)$ surface single positively charged. ${ }^{6,7} \mathrm{We}$
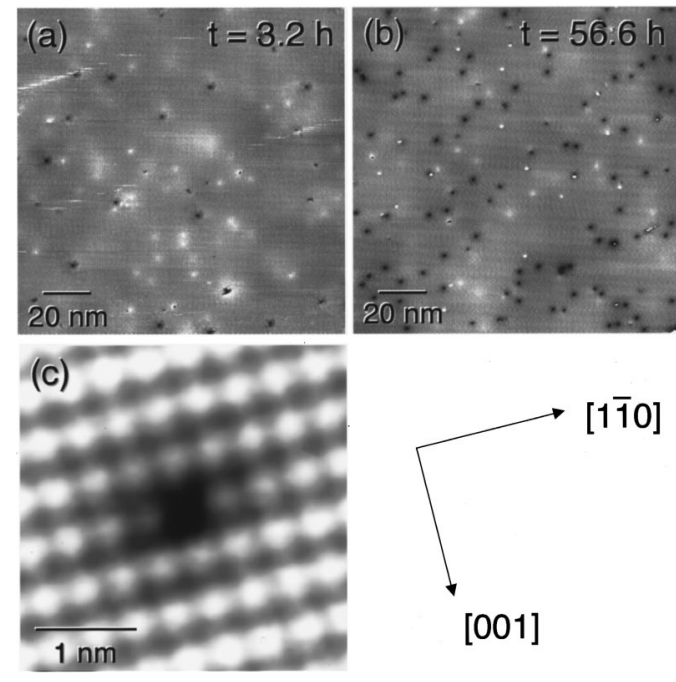

FIG. 1. Constant-current STM images of the occupied states of the cleaved $\operatorname{InP}(110)$ surface (a) 3.2 and (b) $56.6 \mathrm{~h}$ after cleavage. The concentration of $P$ vacancies appearing as dark contrast features increases with time. (c) shows a high resolution STM image of the occupied density of states acquired at $-2.3 \mathrm{~V}$ and $0.4 \mathrm{nA}$ current. Frame (a) and (b) were acquired at -3.0 and $-2.2 \mathrm{~V}$ tunneling voltage and 0.6 and $0.4 \mathrm{nA}$ current, respectively. The sample had a carrier concentration of $1.1 \times 10^{18} \mathrm{~cm}^{-3}$. 


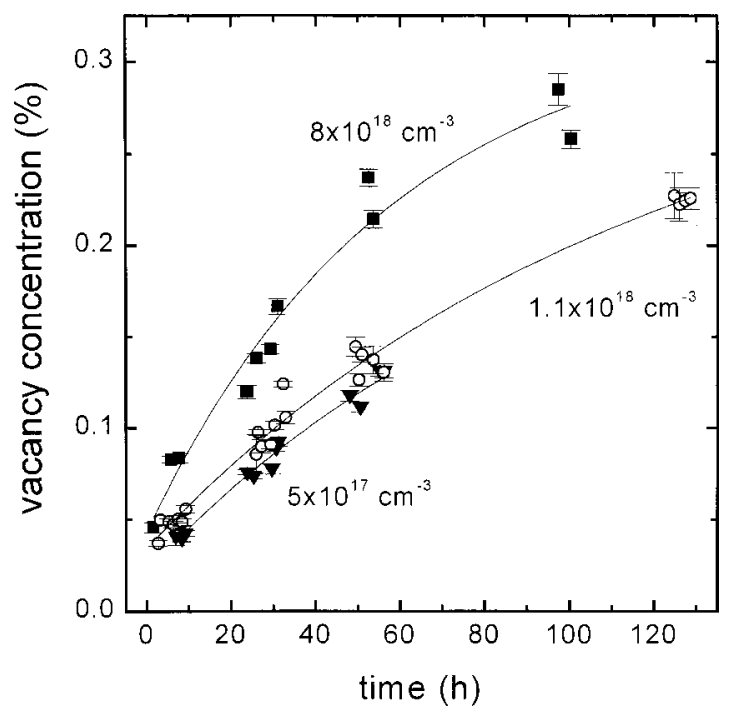

FIG. 2. Concentrations of $P$ vacancies in fraction of $P$ surface lattice sites as a function of time after cleavage at room temperature. Shown are the data sets for three $\operatorname{InP}(110)$ surfaces with different carrier concentrations. The solid lines should guide the eye.

found that only the concentration of $\mathrm{P}$ vacancies increases in STM images obtained at increasing times after cleavage. We can exclude that the tip of the STM produces vacancies, because we acquired the STM images with tunneling conditions, under which the frequency of tip-induced migration and formation events is negligible. ${ }^{4}$ Furthermore, we avoided taking concentration values from STM images showing surface areas, which were repeatedly scanned by the tip. Thus the increase of the vacancy concentration with time is only due to the thermal formation of the vacancies at room temperature.

Figure 2 shows the quantitative vacancy concentration data deduced from the STM images as a function of the time after cleavage for three $\operatorname{InP}(110)$ surfaces with different carrier concentrations. One can recognize three effects: (i) The concentration of the vacancies increases for each sample with time. (ii) The rate of formation of the vacancies (slope of the data) decreases for each data set with increasing time. (iii) The concentration at constant time increases with increasing carrier concentration of the samples. Note that the scattering of the concentration values is due to local inhomogeneities of the dopant concentration (and thus carrier concentration $)^{8}$ and not due to insufficient statistics.

In order to analyze the data, we have to consider the different processes affecting the concentration of surface vacancies: (i) The thermal formation of vacancies increasing the vacancy concentration, (ii) a possible diffusion of surface vacancies into the bulk, and (iii) an annihilation of vacancies by adatoms reducing both the surface vacancy concentration. The diffusion of vacancies into the bulk is negligible, because it only occurs at temperatures above $140{ }^{\circ} \mathrm{C}$. ${ }^{6}$ By taking our data at room temperature we first keep the temperature over long times constant with high accuracy and secondly, we avoid that diffusion into the bulk plays any significant role. On basis of the mechanisms (i) and (ii) the increase of the number of vacancies per time interval $d N_{v} / d t$ is ${ }^{9}$ $d N_{v} / d t$ is $^{9}$
Downloaded 15 Dec 2006 to 134.94.122.39. Redistribution subject to AIP license or copyright, see http://apl.aip.org/apl/copyright.jsp

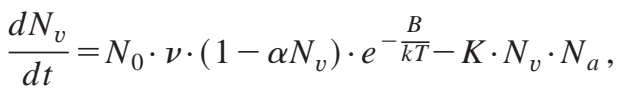

where $\nu$ is the attempt frequency with which an atom attempts to leave its lattice site to form a vacancy, $B$ is the barrier height for the formation of the vacancy, $k$ Boltzmann's constant, $N_{0}$ the number of lattice sites, $K$ the rate coefficient for the adatom-vacancy recombination process, and $N_{a}$ the concentration of adatoms. The factor $\left(1-\alpha N_{v}\right)$ takes into account that the presence of a charged vacancy reduces effectively the lattice sites available for vacancy formation due to a charge repulsion. In our case we concentrated on very low vacancy concentrations, such that vacancy-vacancy interactions are negligible and the factor can be approximated by 1 . A self-consistency test showed that our data is well within this approximation for the vacancy concentrations investigated. Furthermore the low concentration limit reduces the number of adatoms to such a degree that the adatom-vacancy recombination part can be neglected. Note that with these approximations Eq. (1) describes an apparently linear dependence of the vacancy concentration as a function of time. This is, however, not the case, since the barrier $B$ is not constant, but rather a function of the position of the Fermi energy as deduced below (the Fermi level at the surface in turn is shifted by small concentrations of positively charged surface vacancies).

In order to determine the barrier height $B$ for the formation of the vacancy, we need to know the attempt frequency. It can be approximated by the Debye frequency $(8.8 \mathrm{THz})^{10}$ or by the optical phonon frequency of the $\operatorname{InP}(110)$ surface $(2 \mathrm{THz}){ }^{11,12}$ For the determination of the barrier we extracted the slope of the data $\left(d N_{v} / d t\right)$ by fitting an exponential function to the measured data. Using the slope at infinitely small vacancy concentration $(t \rightarrow 0)$ and a range of $1-10 \mathrm{THz}$ as attempt frequency, we obtain for the highest doped sample a range for the value of the barrier height $B$ of $1.15-1.21 \mathrm{eV}$, respectively.

For the determination of the effect of the carrier concentration on the barrier height we determined the relative changes of the barrier as a function of the position of the Fermi energy at the surface. The discussion in terms of relative changes in barrier height has the advantage that the relative values are not affected by the attempt frequency and thus the largest source of uncertainty is removed. This allows us to probe much smaller variations of the barrier height. Figure 3 shows the relative variations of the barrier height as a function of the position of the Fermi energy on the surface. Three data points (filled squares) were extracted from the data extrapolated to the cleavage time (Fermi energy on the surface equals that in the bulk) and three data points (open circles) were determined for vacancy concentrations of about $0.1 \%-0.15 \%$. In the latter case the vacancies formed on the surface induced a band bending at the surface shifting the band edges relative to the Fermi energy. We calculated the band bending due to vacancies on basis of the data given in Ref. 4. The error bars given indicate the relative temperature uncertainty and the error of the determination of the slope. All data points suggest a linear dependence of the barrier height for vacancy formation on the position of the Fermi 


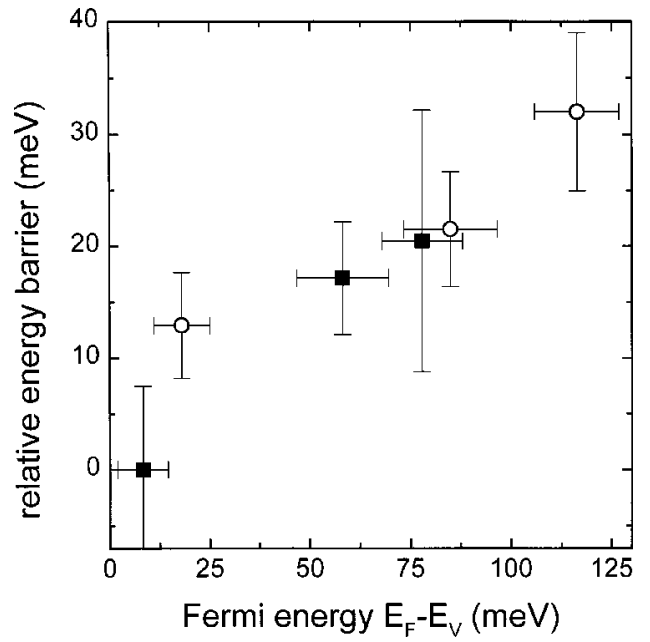

FIG. 3. Relative changes of the barrier height for the formation of positively charged $\mathrm{P}$ vacancies on $p$-doped $\operatorname{InP}(110)$ surfaces as a function of the position of the Fermi energy on the surface. Filled squares show the data for surface Fermi level positions equal to those in the bulk (limit of zero vacancy concentration) and open circles show data for Fermi level positions determined by the bulk and by the surface band bending induced by the presence of positively charged surface $\mathrm{P}$ vacancies.

points shown as open circles (values with surface band bending) with those shown as filled squares (no band bending at the surface), shows that the barrier is only affected by the position of the Fermi energy at the surface and not by that in the bulk crystal.

At this stage we address first the values of the barrier height. To our knowledge no values for the energy barrier height for the formation of a vacancy on a (110) surface of a compound semiconductor has been calculated so far. We thus can only check the consistency of our results with the theoretical calculations by comparing our barrier with the calculated formation energy. For this we have to recall that the barrier height is always larger than or equal to the formation energy. Indeed, calculations yielded formation energies of $1 \mathrm{eV}$ for the positively charged $\mathrm{P}$ vacancy on $\mathrm{P}$ rich, $p$-doped $\operatorname{InP}(110)^{13}$ consistent with our results. For further comparison, we can only use diffusion barriers of vacancies on the related GaAs(110) surface of 1.3 and $1.5 \mathrm{eV} .^{14,15}$ If we take into account that the bond strength of InP is weaker than that of GaAs, our vacancy formation barrier compares reasonably well with the diffusion barriers on GaAs.

For the discussion of the origin of the dependence of the barrier height on the Fermi energy one has to recall that the vacancies formed on the surfaces are positively charged. In order to form a charged vacancy it is necessary to separate electrical charges. This charge separation is likely to occur simultaneously or shortly after the formation and relaxation of the vacancy. In order to understand how a charge separation can affect the barrier, we recall that the formation energy of a positively charged defect increases linearly with the Fermi energy moving toward midgap, since the extracted electron has to be shifted above the Fermi energy. In analogy if a charge separation occurs at the time the vacancy formation process reaches the maximum barrier height along its reaction path, the barrier height will be a function of the position of the Fermi energy. The observed increase of the barrier height with the Fermi energy moving toward midgap is consistent with the direction of change expected for a positively charged defect. Thus the observed dependence of the barrier height can be interpreted as the signature of a charge separation taking place at the time when the intermediate defect configuration reached the maximum barrier height along the reaction path.

In conclusion, we demonstrated a methodology to determine the barrier height for the formation of defects on surfaces using scanning tunneling microscopy. We found that the rate of formation of positively charged $\mathrm{P}$ vacancies on $\operatorname{InP}(110)$ increases with increasing charge carrier concentration of the samples. A quantitative analysis of the data yields a barrier in the range of $1.15-1.21 \mathrm{eV}$. The barrier height increases when the Fermi level moves toward midgap. This functional dependence indicates a charge separation occurring simultaneously with the mechanical removal process of the atom from its lattice site. The determination of quantitative values for barrier heights as shown here allows to describe the kinetics of formation and thus predict the concentrations of charged defects on semiconductor surfaces. An analogous determination of barrier heights of defects in semiconductor growth surfaces may help to optimize conditions for handling or growth of device structures.

The authors thank K. H. Graf for technical support and G. Schwarz for helpful discussions.

${ }^{1}$ K. Schroeder, B. Engels, P. Richard, and S. Blügel, Phys. Rev. Lett. 80, 2873 (1998)

${ }^{2}$ A. Kley, P. Ruggerone, and M. Scheffler, Phys. Rev. Lett. 79, 5278 (1997).

${ }^{3}$ M. B. Johnson, O. Albrektsen, R. M. Feenstra, and H. W. M. Salemink, Appl. Phys. Lett. 63, 2923 (1993); 64, 1454 (1994).

${ }^{4} \mathrm{Ph}$. Ebert, Surf. Sci. Rep. 33, 121 (1999).

${ }^{5} \mathrm{Ph}$. Ebert, K. Urban, and M. G. Lagally, Phys. Rev. Lett. 72, 840 (1994).

${ }^{6} \mathrm{Ph}$. Ebert, M. Heinrich, M. Simon, C. Domke, K. Urban, C. K. Shih, M. B. Webb, and M. G. Lagally, Phys. Rev. B 53, 4580 (1996).

${ }^{7}$ K.-J. Chao, A. R. Smith, and C. K. Shih, Phys. Rev. B 53, 6935 (1996); Ph. Ebert, X. Chen, M. Heinrich, M. Simon, K. Urban, and M. G. Lagally, Phys. Rev. Lett. 76, 2089 (1996).

${ }^{8}$ Ph. Ebert, T. Zhang, F. Kluge, M. Simon, Z. Zhang, and K. Urban, Phys. Rev. Lett. 83, 757 (1999).

${ }^{9}$ J. Philibert, Atom Movements, Diffusion, and Mass Transport in Solids (Les Editions de Physique, Les Ulis, 1991).

${ }^{10}$ J. C. Brice, in Properties of Indium Phosphide (INSPEC, London, 1991), p. 18.

${ }^{11}$ U. Schroeder, J. Fritsch, and P. Pavone, Physica B 219/220, 434 (1996).

${ }^{12}$ H. Nienhaus and W. Mönch, Surf. Sci. 328, L561 (1995).

${ }^{13} \mathrm{G}$. Schwarz (private communication).

${ }^{14}$ R. J. Pechman, X.-S. Wang, and J. H. Weaver, Phys. Rev. B 51, 10929 (1995).

${ }^{15}$ G. Lengel, M. Weimer, J. Gryko, and R. E. Allen, J. Vac. Sci. Technol. A 12, 1855 (1994) 